\title{
Generation of diffractive optical elements onto a photopolymer using a liquid crystal display
}

\author{
A. Márquez $z^{*, 1,3}$, S. Gallego ${ }^{1,3}$, M. Ortuño ${ }^{1,3}$, E. Fernández ${ }^{2,3}$, \\ M. L. Álvarez ${ }^{1,3}$, A. Beléndez ${ }^{1,3}$, I. Pascual ${ }^{2,3}$ \\ ${ }^{1}$ Dept. de Física, Ing. de Sistemas y Teoría de la Señal, Universidad de Alicante, P.O. Box 99, E- \\ 03080, Alicante, Spain \\ ${ }^{2}$ Dept. de Óptica, Farmacología y Anatomía, Universidad de Alicante, P.O. Box 99, E-03080, \\ Alicante, Spain \\ ${ }^{3}$ I.U. Física Aplicada a las Ciencias y las Tecnologías Universidad de Alicante, P.O. Box 99, E- \\ 03080, Alicante, Spain
}

\begin{abstract}
Liquid crystal displays (LCDs) are widely used as spatial light modulators (SLMs) in many applications (optical signal processing, holographic data storage, diffractive optics...). In particular, as an alternative microoptics recording scheme we have explored the possibility to use a LCD to display the diffractive optical element (DOE) to be recorded onto a photosensitive phase material, so as to enhance the flexibility of the recording architecture. In this application the LCD acts as an amplitude dynamic transparency. By means of an optical system we image the function addressed to the LCD onto the recording material. The element to be recorded onto the phase material can be easily changed simply by changing the function addressed to the LCD. Among the recording materials, photopolymers provide very attractive capabilities. They present a great flexibility in their composition, the recording layer can be manufactured in a wide range of possible thicknesses, and they are inexpensive. These properties make it an interesting material to generate the phase DOEs. Both the composition and the thickness need to be optimized for the application to DOEs. In this work we explore the results dealing with the calibration of the recording setup and the photopolymer material. We also analyse the performance of phase-only diffractive lenses generated onto the photopolymer. Promising results have been obtained, where the focalization of the diffractive lenses generated has been demonstrated.
\end{abstract}

Keywords: liquid crystal display, photopolymer, diffractive optical element, spatial light modulator, imaging system, low spatial frequency

\section{INTRODUCTION}

Liquid crystal displays (LCDs) are widely used as spatial light modulators (SLMs) in many applications (optical signal processing, holographic data storage, diffractive optics...). In diffractive optics, LCDs are used to display programmable DOEs [1]. Programmable DOEs are needed in applications where it is interesting an active control through a computer of the function displayed onto the LCD. However, in many cases, passive DOEs are actually needed and a number of microoptics fabrication technologies [2][4][3], such as lithography, diamong turning, direct laser writing, are available to produce high quality passive DOEs. Low cost schemes have also been proposed and analyzed in the literature, such as production of computer-generated phase holograms using graphic devices and contact copying techniques [5]. This technique involves the manufacture of binary absorption masters by high resolution graphic devices. In order to increase the light efficiency, a contact print of these masters was made on a phase material [5]. In this sense, it is possible to use a LCD to display the DOE to be recorded onto the phase material, so as to enhance the flexibility of the recording architecture. In this microoptics application the goal is to use the LCD as an amplitude dynamic transparency. By means

Optical Modelling and Design, edited by Frank Wyrowski, John T. Sheridan, Jani Tervo, Youri Meuret, Proc. of SPIE Vol. 7717, 77170D · C 2010 SPIE · CCC code: 0277-786X/10/\$18 - doi: 10.1117/12.854786 
of an optical system we image the function addressed to the LCD onto the recording material. The element to be recorded onto the photopolymer can be easily changed simply by changing the function addressed to the LCD.

Photopolymer based recording materials have been widely studied for holographic applications[6] [7][8][9] Their good properties as high diffraction efficiencies, low noise, self-developing, easy preparation, high thickness, low cost, etc., make these holographic recording materials optimum candidates for many holographic devices such as holographic memories. Among the different photopolymer compositions, polyvinyl-alcohol/acrylamide (PVA/AA) materials is one of the promising materials in these applications and they have been deeply analyzed in holography [10][11][12] and data storage [13].

In the last years, some studies have been carried out dealing with the recording of very low spatial frequencies (less than 10 lines/mm) in PVA/AA based layers. In Ref. [14][5] a contact-copying process was used to transfer low spatial frequency diffractive optical elements (DOEs) from binary amplitude masks [5] onto the photopolymer, with good results. The same technique was used to analyze the suitability of a wide range of holographic recording materials at low spatial frequencies so as to register DOEs and optical correlation filters [15]. Recently, we proposed a hybrid opticaldigital setup incorporating a liquid crystal display (LCD) to generate phase DOEs onto photopolymers [16]. The application of photopolymers to DOEs generation requires the characterization of the modulation properties of the material. The interferometric techniques proposed in Ref. [17][18] are well adapted for this characterization, since they measure the phase-shift modulation in the range of the very low frequencies usually registered in DOEs.

In this work we explore the additional capabilities offered by the introduction of a LCD to produce DOEs onto a photosensitive material. In particular we consider polyvinyl alcohol (PVA) based photopolymers as the photosensitive material. They present a great flexibility in their composition, the recording layer can be manufactured in a wide range of possible thicknesses, and they are inexpensive. These properties make it an interesting material to generate the phase DOEs. Both the composition and the thickness need to be optimized for the application to DOEs. We consider the inclusion of a cross-linker monomer [N,N_-methylenebisacrylamide (BMA)]. This monomer is used to achieve higher values of refractive index modulation and to obtain more stable gratings [17][19]. In Section 2 we introduce the calibration procedure, interferometric based technique, used to measure the phase-shift versus exposure in the photopolymer layer. We also introduce the general guideline for the calibration procedure to obtain the complex amplitude transmittance versus applied voltage for the LCD. From these results we can propose a combined photopolymer-LCD exposure schedule so as to imprint the necessary phase-shift onto the photopolymer. In Section 3 we explain the experimental setup used to register the DOEs onto the photopolymer and measure its performance in real time. We use the $532 \mathrm{~nm}$ wavelength for recording and the $633 \mathrm{~nm}$ for monitoring. In this work we have generated diffractive lenses onto the photopolymer.

\section{CALIBRATION OF THE LCD AND THE PHOTOPOLYMER}

In this Section we describe the calibration procedure and results obtained both for the LCD and for the photopolymer. First we describe the interferometric setup we use to measure in transmission the phase-shift versus exposure for the photopolymer. Then we describe the calibration results for the intensity transmission versus gray level (applied voltage) for the LCD. These two calibrations are combined to assign the appropriate gray levels onto the LCD to obtain the required phase-shift values onto the photopolymer.

\subsection{Photopolymer layers and interferometric phase-shift calibration}

In Figure 1 we show the experimental setup in transmission geometry to measure the phase-shift as a function of the exposure energy for the photopolymer. This technique was proposed in Ref. [17] for in transmission characterization, and later in Ref. [18] for in reflection characterization. The setup has two arms with an angular separation, one to expose the recording material, whereas the second arm is the interferometer used to measure, in real-time, the phase-shift. The recording material is perpendicularly oriented with respect to the interferometer axis in order to ease the analysis of the interferometric results: at an oblique incidence we should take into account both the Fresnel coefficients at the interface and the increase of distance in the propagation across the layer. 
In the first arm, the exposure beam provided by a solid-state Nd-YVO4 Verdi laser with a wavelength of $532 \mathrm{~nm}$ (at this wavelength the dye presents the maximum absorption) is expanded and collimated using a spatial filter and a lens, obtaining a beam with $1.5 \mathrm{~cm}$ of radius. A wave plate and a neutral filter (attenuator) are added before the spatial filter to control the orientation and the intensity of the linearly polarized beam produced by the laser Nd-YVO4. A polarizer (P), with its transmission axis oriented along the vertical of the lab, is introduced to produce a beam with TE polarization incident onto the recording material. This incident beam forms a certain angle with respect to the photopolymer layer. We adjust the laser power so that the exposure intensity that impinges on the layer is $0.4 \mathrm{~mW} / \mathrm{cm}^{2}$ (this is the value corrected from the Fresnel coefficient at the air-photopolymer interface at an incidence of $30^{\circ}$ for TE polarization). A half-opened diaphragm is used to leave an unexposed area in the photopolymer layer. Afterwards, when we register the DOEs in the photopolymer, we will adjust the exposure intensity onto the material so that we obtain $0.4 \mathrm{~mW} / \mathrm{cm}^{2}$ when the LCD is addressed with the largest intensity transmission value.

In the interferometric arm, to generate the interferences pattern we use a He-Ne laser, since the photopolymer does not present any absorption at $633 \mathrm{~nm}$. We have implemented a Young's fringes based two beams interferometer. We use a grating with a spatial frequency of 4 lines $/ \mathrm{mm}$ to generate a series of diffracted orders from the unexpanded $\mathrm{He}-\mathrm{Ne}$ beam; we block all the orders except -1 and +1 . One of the two orders impinges on the exposed zone (illuminated by the $\mathrm{Nd}-\mathrm{YVO} 4$ laser) and the other one impinges on the non-exposed zone. The distance between the two orders is about 1 $\mathrm{cm}$, so as to eliminate the influence of the monomer diffusion in the polymerization process. Once the two orders have propagated throughout the photopolymer, a lens is used to make them interfere. A microscope objective is used to amplify the interference pattern onto a CCD camera. This pattern is captured in real-time as a function of exposure at specific time intervals.

We note that afterwards, we design the DOEs to be used with the $633 \mathrm{~nm}$ wavelength. The LCD will be illuminated with the $532 \mathrm{~nm}$, and this is the wavelength used to expose the photopolymer.

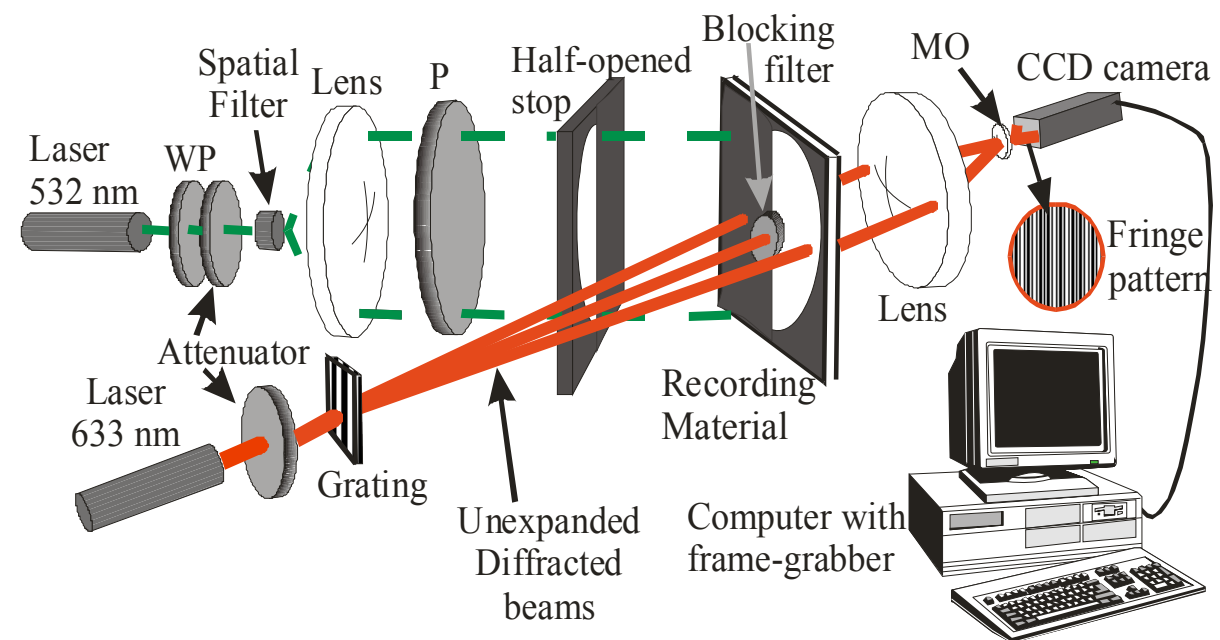

Figure 1. Experimental setup. The recording material is exposed with the green laser beam $(\lambda=532 \mathrm{~nm})$ and the phase-shift is measured with the red beam $(\lambda=633 \mathrm{~nm})$. $\mathrm{P}$ is polarizer, WP is wave plate, MO is microscope objective.

We have considered thin layers about $100 \mu \mathrm{m}$ thick with the basic chemical composition using acrylamide monomer (AA) and with the inclusion of a cross-linker monomer [N,N_-methylenebisacrylamide (BMA)]. The formulation we use has been proved to provide $360^{\circ}$ phase-shift depth [17], that is what is needed to record multilevel phase DOEs. In principle, the BMA is necessary to achieve higher values of refractive index modulation, thus to be able to reach $360^{\circ}$ phase depth without increasing too much the thickness of the layer, which could introduce material inhomogeneities, longer drying times. Furthermore, BMA promotes more stable gratings [19]. We consider PVA with a molecular weight of 130000 , and TEA concentration $0.4 \mathrm{M}$ (see Table 1). The photopolymerizable solution is prepared mixing yellowish eosin (YE, the dye), together with a mixture of acrylamide (the monomer) and triethanolamine (the co-initiator), and adding the PVA (the binder). The solutions are prepared using a conventional magnetic stirrer, under red light and in standard laboratory conditions (temperature, pressure, relative humidity). The solutions are deposited, by gravity, over 
glasses (size 20x40 cm2). Afterwards the solutions are left in the dark to allow the water evaporation. When a high percentage of the water content has already evaporated, the material is cut into squares $\left(6.5 \times 6.5 \mathrm{~cm}^{2}\right)$ using a glass cutter. The water solution used in this paper is presented in Table 1.

Table 1. Water solutions used to obtain phopopolymerizable "dry" layers.

\begin{tabular}{|l|ll|}
\hline Component & \multicolumn{2}{|l|}{ Quantity } \\
\hline PVA $\mathbf{M}_{\mathrm{w}}=\mathbf{1 3 0 0 0 0}$ & $8.5 \%$ & $\mathrm{w} / \mathrm{V}$ \\
\hline TEA & 0.4 & $\mathrm{M}$ \\
\hline YE & $2.5 \times 10^{-4}$ & $\mathrm{M}$ \\
\hline AA & 0.40 & $\mathrm{M}$ \\
\hline BMA & 0.05 & $\mathrm{M}$ \\
\hline
\end{tabular}

In Figure 2 we show the results for a typical calibration of phase-shift versus exposure for the photopolymer layer. We observe that we reach a $360^{\circ}$ phase depth for an exposure of about $110 \mathrm{~mJ} / \mathrm{cm}^{2}$. In principle this is what is needed to register multilevel phase-only DOEs. We note that the phase-shift calibration obtained using the interferometric-based setup is a zero frequency limit characterization [17], in which the diffusion processes in the material do not play any role in the index modulation formation. Actually, when recording DOEs, the monomer gradients due to photopolimerization will generate diffusion of the different species in the layer, thus the phase-shift calibration in Figure 2 can be considered as a reasonable starting point but not as an exact calibration. The effect of diffusion has been characterized in previous works dealing with the recording of binary diffractive gratings. Another effect which will be introduced by diffusion processes is that there can be in dark evolution of the index modulation. That is, once the exposure is stopped, it can still be measured a time evolution in the dark of the diffracted and transmitted efficiencies as for example we measured in Ref. [20][21][22] with the recorded binary diffractive gratings. When properly characterized the in dark evolution can be used to calculate in which point exposure should be stopped taking into that afterwards diffusion processes may lead to the a desired index modulation depth value.

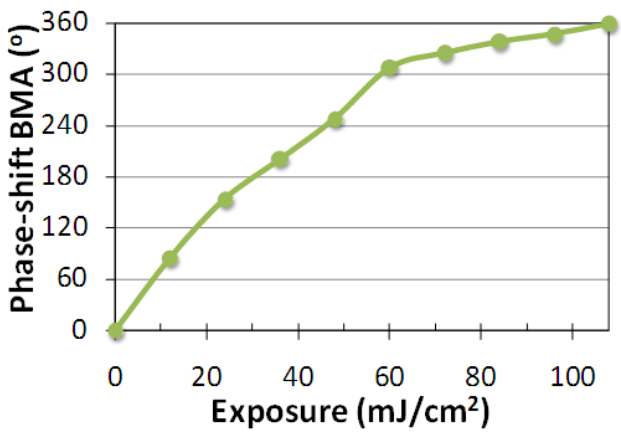

Figure 2. Phase-shift vs. exposure for the photopolymeric material. A phase depth of $360^{\circ}$ is achieved, necessary to use the material to record phase DOEs. We show both the experimental and the fitted data. Incident intensity (after Fresnel correction for TM polarization): $0.4 \mathrm{~mW} / \mathrm{cm}^{2} . \lambda=633 \mathrm{~nm}$.

\subsection{LCD characterization}

In this work, we use a Sony LCD model LCX012BL, extracted from a video projector Sony VPL-V500. The Sony LCX012BL is a $3.3 \mathrm{~cm}$ diagonal active matrix thin film transistor (TFT) panel, with VGA resolution (640 x 480 pixels). The pixels are square with a pixel center to center separation of $41 \mu \mathrm{m}$ and a width of $34 \mu \mathrm{m}$. We use the electronics of the video projector to send the voltage to the pixels of the LCD. In the calibration of the LCD we use well established techniques to obtain the values for a series of parameters [23][24]. In Table 2 we show the values for the voltage independent parameters. We must also calibrate the so called voltage dependent parameters [23][24].For the calibration of these values, measurements have been taken using the unexpanded beams for two different wavelengths: $633 \mathrm{~nm}$ from 
a He-Ne laser and the 532 from a Nd: $\mathrm{YVO}_{4}$ laser. Further details about the model used and the calibration procedure can be found in the papers [23][24] and in the references therein.

Table 2.Values of the parameters for the LCD used in the experiments.

\begin{tabular}{|c|c|c|c|}
\hline$\alpha\left({ }^{\circ}\right)$ & $\Psi_{\mathrm{D}}\left({ }^{\circ}\right)$ & $\beta_{\max }(\lambda=\mathbf{6 3 3} \mathbf{~ n m})$ & $\beta_{\max }(\lambda=532 \mathrm{~nm})$ \\
\hline-92 & 47 & 135 & 171 \\
\hline
\end{tabular}

Table 3.Values of the angles of the transmission axis of the input and output polarizers respectively with respect to the director axis in the input and output faces of the LCD.

\begin{tabular}{|c|c|c|}
\hline Modulation Regime & $\boldsymbol{\varphi}_{1}\left(\mathbf{(}^{\mathrm{o}}\right)$ & $\boldsymbol{\varphi}_{2}\left({ }^{\circ}\right)$ \\
\hline Amplitude-only & 46.7 & 49.5 \\
\hline
\end{tabular}

Once all the parameters have been obtained and using the model in which they are based [23][24], we can conduct computer optimizations to find the orientation for the axes of the external polarization elements (polarizers and wave plates) to produce the required modulation regime. In this paper, we need the LCD to act as a gray level transmittance transparency, i.e. we need the LCD to work in the amplitude-only regime. In Fig. 3(a) and 3(b) we show respectively the normalized intensity and the phase-shift curves as a function of the gray level for the optimum amplitude-only modulation that can be achieved with our LCD and for $\lambda=532 \mathrm{~nm}$. These results have been obtained considering only linear polarizers. In Table 3 we give the angles for the orientation of the transmission axes for the input and output polarizers. The circles are the experimental values and the continuous line is the simulation provided by the model. We observe the excellent agreement between experiment and theory. We can say that this is an amplitude-only regime. If we consider the addition of wave plates the phase modulation range can be further decreased [24]. The lowest and the highest transmission values in Fig. 3 occur respectively at gray levels 10 and 210.

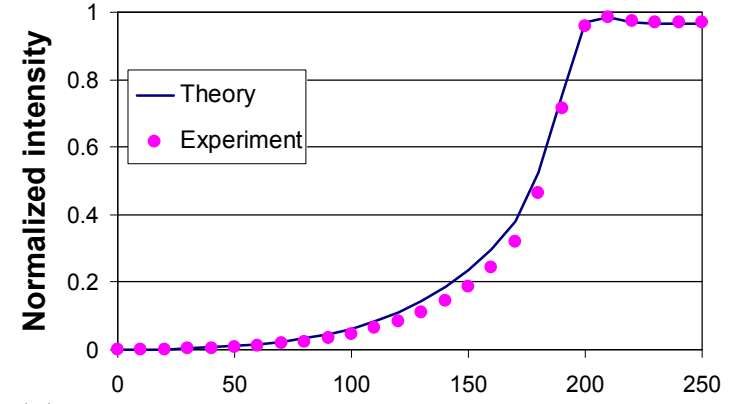

(a)

Gray level

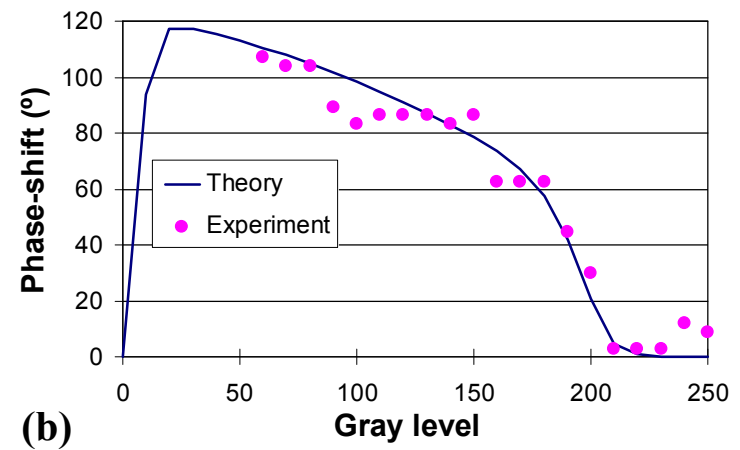

Figure 3. Amplitude-only modulation. (a) Normalized intensity. (b) Phase-shift. The symbols correspond to experimental measurements and the lines correspond to predicted values. $\lambda=532 \mathrm{~nm}$.

In Figure 4(a) we show the intensity transmission curve for the LCD, experimental values and fitting function, that we will be used to register DOEs onto the photopolymer. We consider the gray level interval between 10 and 200 (reaching 210 makes it more difficult to obtain a fitting function). We normalize the intensity transmission at gray level 200, to one.

Using the LCD calibration (Figure 4(a)) and the photopolymer calibration (Figure 2) we obtain the combined calibration curve in Figure 4(b), needed to assign the proper gray level on the LCD in order to produce the desired phase-shift in the photopolymer. The plot in Figure 4(a) considers that the intensity incident onto the photopolymer when the LCD is in its highest transmission value (gray level 200) is $0.4 \mathrm{~mW} / \mathrm{cm}^{2}$. In principle the time needed to achieve a phase-shift depth of $360^{\circ}$ using the values in Fig. 2 should be 240 seconds. As already commented with respect to Figure 2, due to diffusion processes the calibration should not be considered strictly precise but as a good starting point to explore the possibilities of using a combined LCD-photopolymer setup to generate DOEs. 

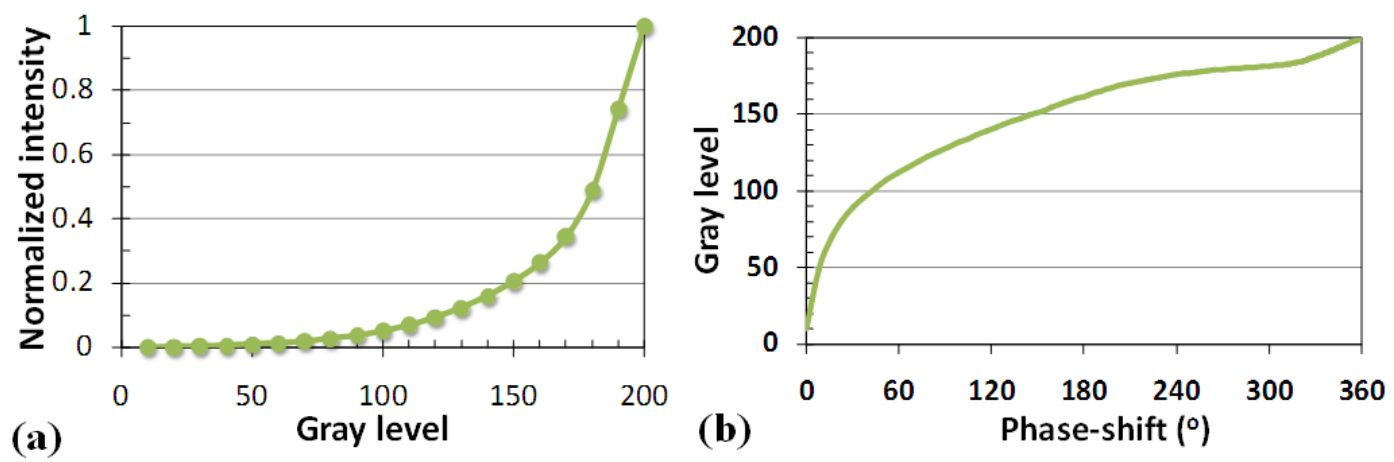

Figure 4. (a) Intensity calibration curve used for the LCD $(\lambda=532 \mathrm{~nm})$. (b) Combined LCD-photopolymer calibration. Gray level addressed to the LCD consider exposing wavelength $\lambda=532 \mathrm{~nm}$, and the phase-shift in the photopolymer considers illumination wavelength $\lambda=633 \mathrm{~nm}$. Intensity incident onto the photopolymer when the LCD is in its highest transmission value (gray level 200) is $0.4 \mathrm{~mW} / \mathrm{cm}^{2}$. The time needed to achieve a phase-shift depth of $360^{\circ}$ using the values is 240 seconds

\section{ANALYSIS OF DIFFRACTIVE OPTICAL ELEMENTS GENERATED}

In previous work we generated binary diffractive gratings with various spatial frequencies [20][21][22]. They served basically to evaluate the fidelity of the phase modulation spatial profile with respect to the desired one and to analyze the diffusion processes in the material. We were able to obtain efficiencies close to the theoretical maximum of $40 \%$ in various cases. Now in this work, we want to analyze the generation of multilevel phase diffractive optical elements. Specifically in this work, we will generate diffractive optical lenses. First we introduce the experimental setup we use, and then we will provide some of the results obtained with the recorded diffractive optical lenses.

\subsection{Experimental set-up to generate and measure in real-time the diffractive optical lenses}

In Figure 5 we show the experimental setup used to register and analyse the diffractive optical lenses. The recording process uses wavelength $532 \mathrm{~nm}$, and the lenses are designed to be used with the $633 \mathrm{~nm}$ wavelength. The system is designed so that it enables to measure the performance of the lenses in real-time while the recording process is taking place. Most of the elements in the setup where already explained in Section 2.

We distinguish two arms: the recording arm, using the wavelength $532 \mathrm{~nm}$ provided by a solid-state Verdi laser (NdYVO4), and the analyzing arm, using the wavelength $633 \mathrm{~nm}$ provided by a He-Ne laser. In the recording arm we place the LCD sandwiched between two polarizers (P), which are oriented according to the values in Table 3, to produce amplitude-mostly modulation. Then, a lens is used to image the intensity transparency displayed on the LCD onto the recording material. The analyzing arm is designed so that the beam of light incident onto the recording material is collimated. Stop 2 is used to limit the aperture of the collimated beam. We need to introduce a non-polarizing, thin beam-splitter so that both beams of light, the recording and the analyzing, follow the same path. After the recording material the recording beam defocuses so that the light incident onto the final CCD camera is only coming from the analyzing beam. The lens recorded on the photopolymer is responsible for the focusing of the $633 \mathrm{~nm}$ wavelength beam. We add a mirror to fold the light path and reduce the extent of the system, and we use a 10X objective microscope to image different planes of the point spread function (PSF) generated by the diffractive lens onto the CCD camera. We use a high dynamic range CCD, which is necessary to appreciate details in the PSF. We use the CCD camera model pco.1600 from pco.imaging. This is a high dynamic14 bits cooled CCD camera system with a resolution of $1600 \times 1200$ pixels, and a pixel size of $7.4 \times 7.4 \mu \mathrm{m}^{2}$. The camera is also used, without the objective microscope, on the plane of the recording material to evaluate the intensity pattern actually imaged from the LCD plane. 


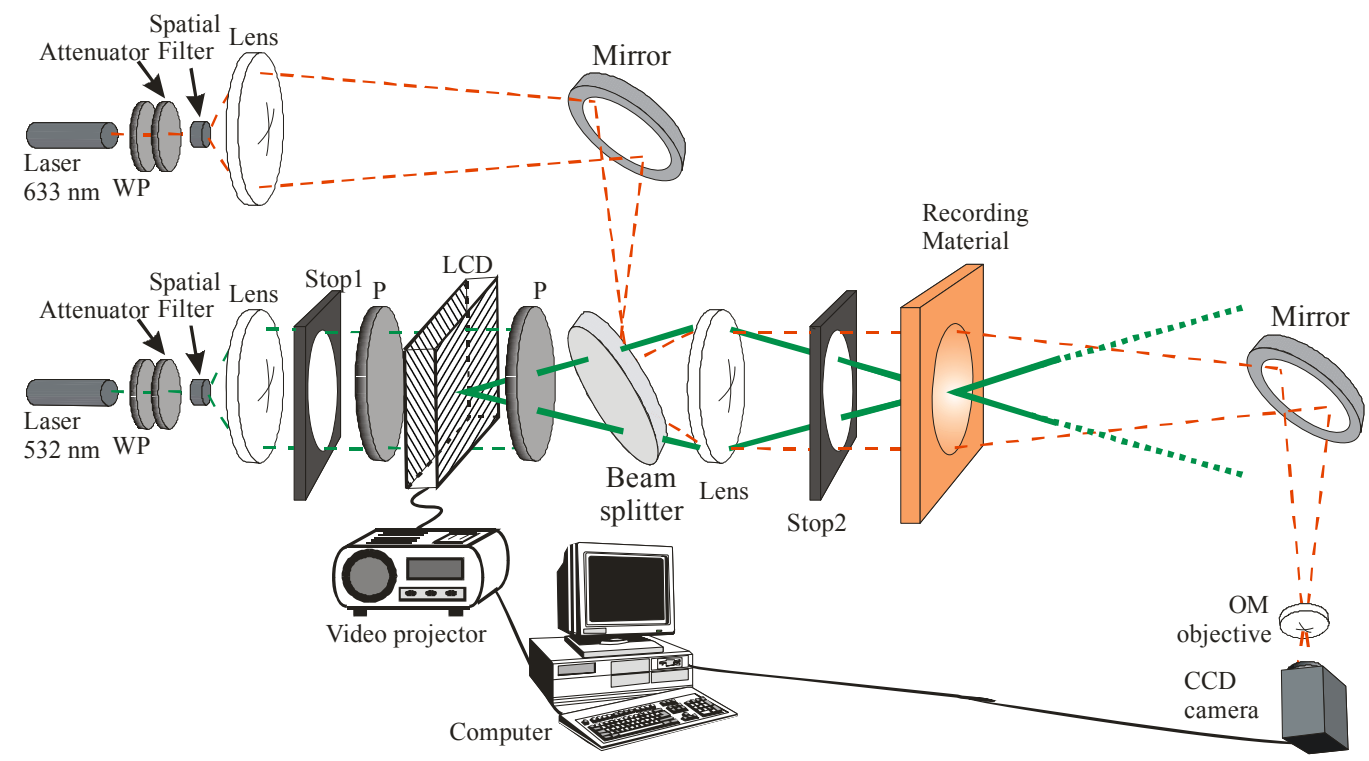

Figure 5. Experimental setup used to register and analyse in real-time the DOEs (diffractive lenses).

The calibration curve described in Figure 4(b) is used to produce the lenses onto the photopolymer. Intensity incident onto the photopolymer when the LCD is in its highest transmission value (gray level 200) is $0.4 \mathrm{~mW} / \mathrm{cm}^{2}$. The actual magnifying factor produced by the objective onto the CCD camera is calibrated using as the reference object a grating whose period is well known: we obtain that the scale on the CCD sensor corresponds to $0.83 \mu \mathrm{m} / \mathrm{pixel}$.

\subsection{Results with phase-only diffractive lenses}

Our goal is to produce a diffractive lens on the photopolymer with a focal of 1 meter. To this goal we calculate the corresponding amplitude pattern to be displayed onto the LCD. We use the calibration in Figure 4, to provide the proper look-up table for the gray levels to be addressed onto the LCD. Onto the LCD what we display is an intensity transparency mask, with the corresponding ring spacing for a diffractive lens of 1 meter focal length.

In Figure 6(a) we show the image obtained using the CCD camera, when located in the recording plane. We see the characteristic ring structure with decreasing period as we move away from the center of the diffractive lens. In Figure 6(b) we show the intensity plot along the horizontal line passing through the center of the lens. This intensity pattern in Figure 6 is the exposure pattern which will be recorded on the photopolymeric material.
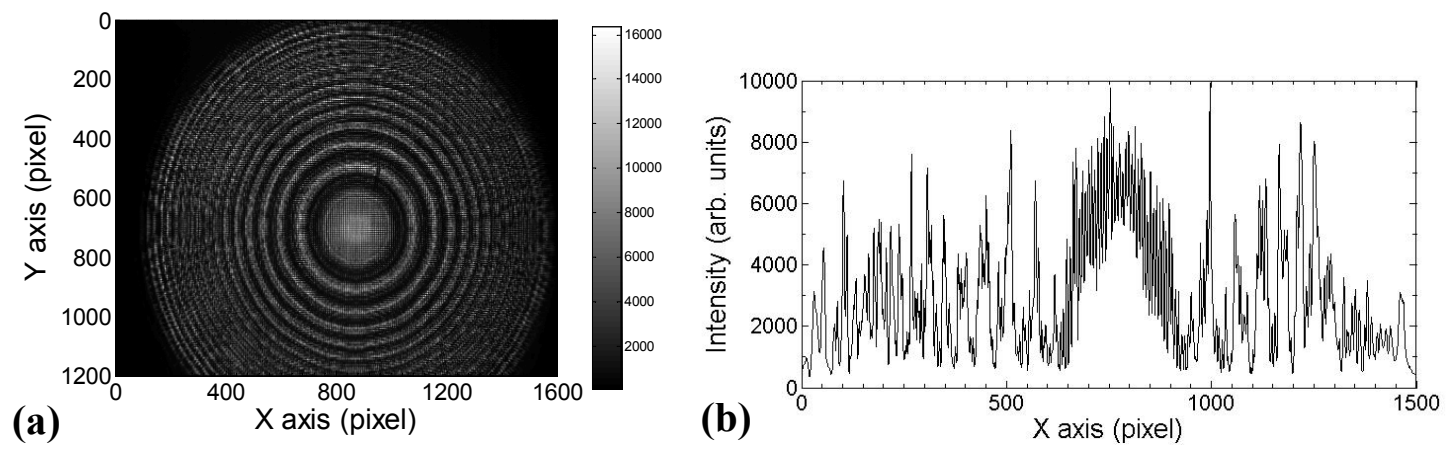

Figure 6. (a) Image of the LCD plane, where the intensity transmittance equivalent lens is displayed, captured by the CCD camera onto the recording plane where the photopolymer should be exposed. (b) Intensity profile across the horizontal line passing through the center of the lens. 
The illuminated area on the LCD is about $8 \mathrm{~mm}$ in diameter. Using Figure 6(a) we obtain that the actual aperture of the lens onto the recording plane is about $11 \mathrm{~mm}$. That is, the imaging system is producing a magnifying factor about 1.4. Taking this into account we may calculate what is the actual focal length of the lens recorded on the photopolymer. A lens with a focal length $f$, has a phase profile given by [4],

$$
\varphi=\frac{\pi r^{2}}{\lambda f}
$$

, where $\lambda$ is the illumination wavelength, and $r$ is the radial coordinate (in the LCD plane). In the case of a diffractive lens, the phase profile introduced is obtained from equation (1) applying modulo $2 \pi$ radians [4]. If now the aperture of the lens is magnified (or demagnified) by a factor $\kappa$, then we may say that,

$$
r^{\prime}=\kappa r
$$

, where $r$ ' is the radial coordinate in the image plane (where the recording material is located). The total phase depth stays the same, thus,

$$
\varphi^{\prime}=\frac{\pi r^{\prime 2}}{\lambda f^{\prime}}
$$

and $\varphi_{M A X}^{\prime}=\varphi_{M A X}$. Then, the actual focal length $f^{\prime}$ in the lens in the recording plane has to be,

$$
f^{\prime}=\kappa^{2} f
$$

This means that if the focal length implemented in the amplitude transmittance equivalent lens onto the LCD is $f=1 \mathrm{~m}$, then the actual focal length recorded in the phase diffractive lens in the photopolymer is $f^{\prime}=2 \mathrm{~m}$, considering a magnifying factor of $\kappa=1.4$. Actually in the lenses we have recorded in the photopolymer we have found very clearly focalization at a distance from the recording plane $z=0.68 \mathrm{~cm}$, which is one third of the predicted focal. Thus it could actually be the focalization due to one of the secondary focal planes introduced by the diffractive lens. At longer distances it was not so clear that we had focalization, and actually because of room limitations we could not verify that there was focalization at two meters of distance. In future work we will try to clarify this point.

In Figure 7 we show the images captured at the best image plane $(z=68 \mathrm{~cm})$ (Figure 7(c)) and at two defocused planes before (Figure 7(a) and 7(b)) and at two defocused planes after (Figure 7(e) and 7(f)) the best image plane. In Figure 7(d) we plot the intensity profile along the horizontal and vertical lines passing through the peak intensity point in the best image plane. We note that the images in the defocused planes are less energetic (see the gray level bar on the right of each figure) than the best image plane image. If we go further away from the best image plane the peak intensity decreases till the central minimum disappears. We note that the images (a-c) present a relatively good circular symmetry. However, this symmetry disappears in images (e-f) after the best image plane, and actually we can appreciate the appearance of a second peak. This could be due to slight misalignment in the illumination of the recorded lens, but also to inhomogeneities in the photopolymer layer which produce an inhomogeneous diffraction efficiency across the diffractive lens and aberrations due to thickness variations. We also note that the two planes before the best image plane have higher peak intensities than the two after, which may also indicate some degree of spherical aberration in the diffractive lens. 

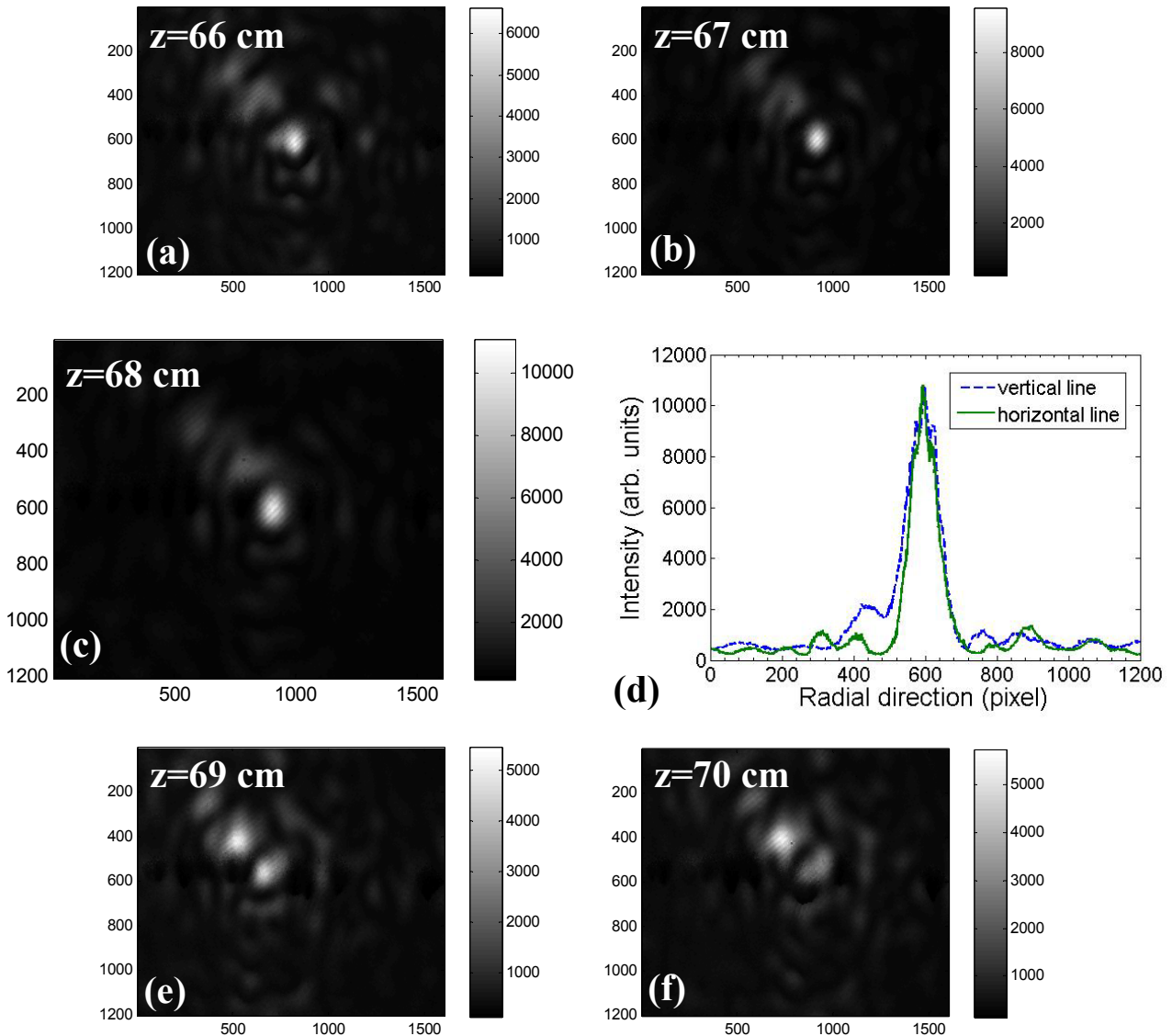

Figure 7. Defocused planes before (a-b) and after (e-f) of the best image plane (c) obtained at a distance $\mathrm{z}=68 \mathrm{~cm}$ of the plane of the diffractive phase lens recorded. In (d) we show the intensity profile along the horizontal and vertical lines passing through the peak intensity point in the best image plane.

In Figure 7(d) we find that the two transversal cuts (horizontal and vertical) generally coincide one each other in the central peak but the sidelobes are not so well overlapped. We may estimate from these cuts that the width of the central peak is about 200 pixels, which taking into account the scale of the image produced by the objective onto the CCD $(0.83$ $\mu \mathrm{m} / \mathrm{pixel})$, makes that the width in lab coordinate is about $170 \mu \mathrm{m}$. Now, we can estimate the theoretical width for the Airy spot in the case of lens with a focal length of $f=0.68 \mathrm{~m}$, illuminated with a wavelength of $\lambda=0.633 \mu \mathrm{m}$, and with an aperture radius of $R=5.5 \mathrm{~mm}$. Using the formula for the width of the Airy spot,

$$
r=\frac{0.61 \lambda f}{R}
$$

, we obtain a radius of $48 \mu \mathrm{m}$, i.e. a diameter of about $100 \mu \mathrm{m}$. Therefore the experimental value is larger. This may indicate some apodization effect in the plane of the lens, which would be due to a non-uniform transmission whose value decreases with the radial coordinate. This non-uniform transmission could be simply due to a decrease in the diffraction efficiency in the outer rings of the diffractive lens with respect to the inner rings. Outer rings are narrower leading to the availability of less pixels in the LCD to reproduce the desired profile. Therefore, there are less phase levels reproduced in the photopolymer. We may think the diffractive lens as a blazed grating whose period decreases with the radial coordinate. Thus in the outer rings we have a blazed grating with a smaller number of phase levels leading to a smaller diffraction efficiency, i.e. less light addressed onto the primary focus. This would be basically the same effect reported in Ref. [25]for diffractive lenses generated onto LCDs working in the phase-only regime. 


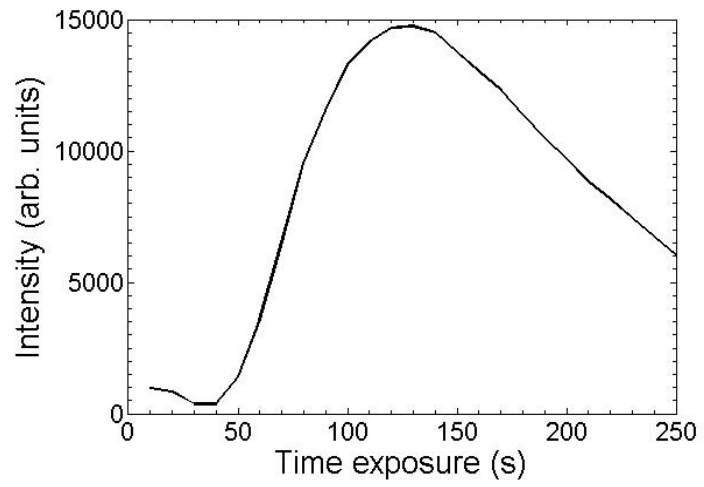

Figure 8 . Intensity value in the center of the best image plane as a function of exposure time. We capture the best image time while the photopolymer is being exposed, taking advantage of the real-time capability of our setup.

Another kind of experiment we have done takes advantage of the real time capability offered by the setup. We place the combined system objective-CCD camera in order to capture the best image plane $(z=68 \mathrm{~cm})$, and we take a picture every ten seconds. In Figure 8 we show the intensity value in the center of the best image plane as a function of time, where we obtain that the maximum is attained after 130 seconds. We define the center of the image as the peak intensity pixel in the image obtained after 130 seconds of exposure. This value, 130 seconds, is smaller than the one expected from the calibration in Section 2, i.e. 240 seconds. This difference is due to variations in the environmental conditions (temperature and humidity) during the drying process of the photopolymer layer, may also be affected by thickness variations between each batch of photopolymer produced, and it is also influenced by the moment in which the photopolymer is used once we have a dried layer: as time passes the material loses sensitivity. Greater control could be obtained if the environmental conditions in the lab could be more stable. In Figure 9(a) and (b) we plot respectively the vertical and horizontal cuts along the center pixel of the best image plane. Both in Figure 9(a) and (b) we plot the intensity profiles for 3 different exposure times. We see that the width of the central peak does not change appreciably among these exposure times.
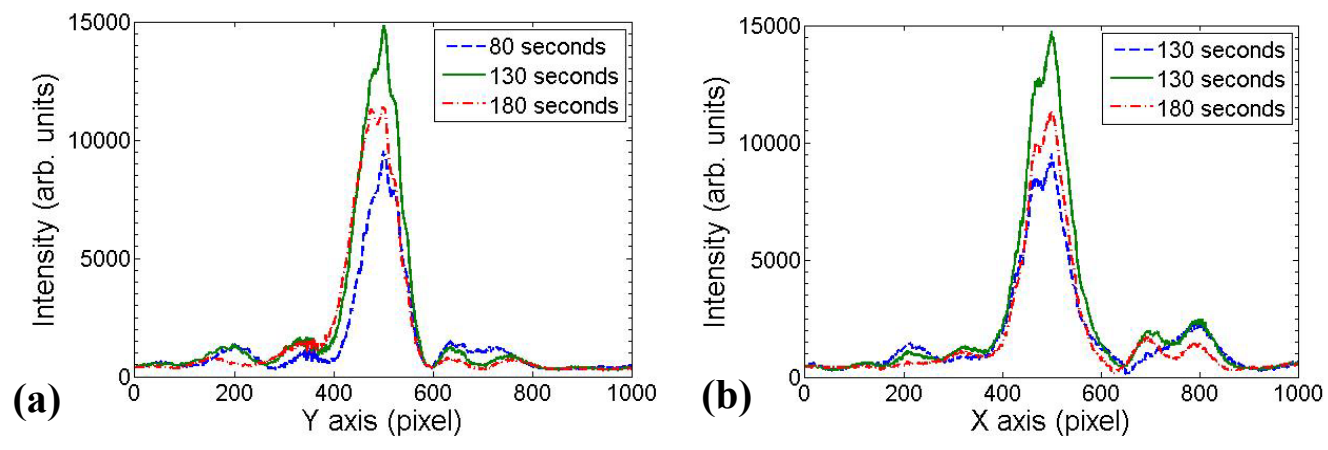

Figure 9. Vertical (a) and horizontal (b) intensity profiles along the center pixel of the best image plane and for 3 different exposure times.

\section{CONCLUSIONS}

In this work we have explored the application of a combined setup using a LCD and a photopolymer material to register multilevel phase diffractive lenses. In this application the LCD acts as an amplitude dynamic transparency. By means of an optical system we image the function addressed to the LCD onto the recording material. The element to be recorded onto the phase material can be easily changed simply by changing the function addressed to the LCD. In this work we 
have presented the results dealing with the calibration of the recording setup with the LCD and the photopolymer material. We have also analysed the performance of diffractive lenses generated onto the photopolymer. We have introduced a real-time setup allowing for the recording of the diffractive lenses, while monitoring its performance. We have successfully recorded diffractive lenses showing a good focalization capability. Possibly thickness inhomogeneities, diffraction efficiency decrease with the radial coordinate (leading to an equivalent apodizing filter onto the plane of the LCD), are some possible causes decreasing the quality of the diffractive lenses generated. Future work will try to address these issues.

\section{ACKNOWLEDGEMENTS}

This work was supported by the "Ministerio de Edicación y Ciencia" (Spain) under projects projects FIS2008-05856C02-01 and FIS2008-05856-C02-02, by the Generalitat Valenciana (projects ACOMP/2009/150, ACOMP/2009/160 and GVPRE/2008/137).

\section{REFERENCES}

[1] A.Márquez, C. Iemmi, J. Campos and M. J. Yzuel, "Achromatic diffractive lens written onto a liquid crystal display"Opt. Lett., 31, 392-394, 2006.

[2] H. P. Herzig, ed., Micro-optics-Elements, Systems, and Applications, (Taylor \& Francis, London, 1997)

[3] J. Turunen \& F. Wyrowski, Diffractive optics for industrial and commercial applications, Akademie Verlag, Berlin (1997).

[4] D. C. O'Shea, T. J. Suleski, A. D. Kathman and D. W. Prather, Diffractive Optics: design, fabrication and test, SPIE (2004).

[5] A. Márquez, J. Campos, M. J. Yzuel, I. Pascual, A. Fimia and A. Beléndez, "Production of computer-generated phase holograms using graphic devices: application to correlation filters", Opt. Eng. 39, 1612-1619 (2000).

[6] A. Pu, D. Psaltis, "High-density recording in photopolymer-based holographic three-dimensional disks", Appl. Opt. 35, 2389-2398, (1996).

[7] Y. Tomita, K. Furushima, K. Ochi, K. Ishizu, A. Tanaka, M. Ozawa, M. Hidaka, and K. Chikama, "Organic nanoparticle (hyperbranched polymer)-dispersed photopolymers for volume holographic storage," Appl. Phys. Lett. 88, 071103 (2006).

[8] A. Márquez, C. Neipp, S. Gallego, M. Ortuño, A. Beléndez and I. Pascual, "Edge enhanced imaging using PVA/acrylamide photopolymer gratings", Opt. Lett. 28(17), 1510-1512 (2003).

[9] S. M. Schultz, E. N. Glytsis, and T. K. Gaylord, "Design of high-efficiency volume gratings couplers for line focusing," Appl. Opt. 37, 2278-2287 (1998).

[10] C. Neipp, A. Beléndez, S. Gallego, M. Ortuño, I. Pascual and J. T. Sheridan, "Angular responses of the first and second diffracted orders in transmission diffraction grating recorded on photopolymer material," Optics Express $11,1835-1843$ (2003).

[11] J. V. Kelly, F. T. O' Neill, C. Neipp, S. Gallego and M. Ortuño and J. T. Sheridan, "Holographic photopolymer materials: non-local polymerisation driven diffusion under non-ideal kinetic conditions" J. Opt. Soc. of Am. B 22, 22, 407-406 (2005).

[12] S. Martin, C. A. Feely and V. Toal, "Holographic recording characteristics of an acrylamide-based photopolymer", Applied Optics 36, 5757-5768 (1994).

[13] M. Ortuño, S. Gallego , C. García , C. Neipp , A. Beléndez and I. Pascual "Optimization of a $1 \mathrm{~mm}$ thick PVA/acrylamide recording material to obtain holographic memories: method of preparation and holographic properties" Applied Physics B, 76, 851-857 (2003).

[14]I. Pascual, A. Márquez, A. Beléndez, A. Fimia, J. Campos and M. J. Yzuel, "Copying low spatial frequency diffraction gratings in photopolymer as phase holograms", J. Mod. Opt. 47, 1089-1097 (2000).

[15] A. Márquez, C. Neipp, A. Beléndez, J. Campos, I. Pascual, M. J. Yzuel and A. Fimia, "Low spatial frequency characterization of holographic recording materials applied to correlation", J. Opt. A: Pure Appl. Opt. 5, S175S182 (2003). 
[16] A. Márquez, S. Gallego, D. Méndez, M. L. Álvarez, E. Fernández, M. Ortuño, C. Neipp, A. Beléndez, I. Pascual, "Application of a liquid crystal display to generate diffractive optical elements onto a photopolymer", Proc. EOS Topical Meeting on Diffractive Optics, 194-195 (2007).

[17] S. Gallego, A. Márquez, D. Méndez, M. Ortuño, C. Neipp, M. L. Alvarez, A. Beléndez, E. Fernández and I. Pascual, "Real-time interferometric characterization of a PVA based photopolymer at the zero spatial frequency limit," Appl. Opt. 46, 7506-7512 (2007).

[18] S. Gallego, A. Márquez, D. Méndez, M. Ortuño, C. Neipp, M. L. Alvarez, A. Beléndez, E. Fernández and I. Pascual, "Analysis of PVA/AA based photopolymers at the zero spatial frequency limit using interferometric methods," Appl. Opt. 47, 2556-2563 (2008)

[19] C. Neipp, S. Gallego, M. Ortuño, A. Márquez, A. Beléndez, I. Pascual "Characterization of a PVA/acrylamide photopolymer. Influence of a cross-linking monomer in the final characteristics of the hologram" Opt. Commun., 224, 27-34 (2003).

[20] S. Gallego, A. Márquez, D. Méndez, C. Neipp, M. Ortuño, A. Beléndez, E. Fernández, I. Pascual, "Direct analysis of monomer diffusion times in polyvinyl/acrylamide materials," Appl. Phys. Lett. 92, 073306 (2008).

[21] S. Gallego, A. Márquez, D. Méndez, S. Marini, A. Beléndez, and I. Pascual, "Spatial phase modulation-based study of PVA/AA photopolymers in the low spatial frequency range," Appl. Opt. 48, 4403-4413 (2009).

[22] S. Gallego, A. Márquez, S. Marini, E. Fernández, M. Ortuño, and I. Pascual, "In dark analysis of PVA/AA materials at very low spatial frequencies: phase modulation evolution and diffusion estimation", Opt. Express 17, 18279- 18291 (2009).

[23] A. Márquez, J. Campos, M. J. Yzuel, I. Moreno, J. A. Davis, C. Iemmi, A. Moreno and A. Robert, "Characterization of edge effects in twisted nematic liquid crystal displays", Opt. Eng. 39, 3301-3307 (2000).

[24] A. Márquez, C. Iemmi, I. Moreno, J. A. Davis, J. Campos and M. J. Yzuel, "Quantitative prediction of the modulation behavior of twisted nematic liquid crystal displays," Opt. Eng. 40, 2558-2564 (2001).

[25] M. J. Yzuel, J. Campos, A. Márquez, J. C. Escalera, J. A. Davis, C. Iemmi and S. Ledesma, "Inherent apodization of lenses encoded on liquid crystal spatial light modulators", Appl. Opt. 39, 6034-6039 (2000). 